\title{
Impacto de la violencia e inseguridad en la competitividad de los estados mexicanos
}

\section{The impact of violence and insecurity on the competitiveness of mexican states}

\author{
Rigoberto Soria-Romo*
}

\begin{abstract}
In this paper, we seek to determine the impact of violence and public insecurity on the competitiveness of the states in México. The starting point is the definition of competitiveness given by Imco. Panel data is applied to three models with different dependent variables: gross fixed capital formation, human talent and Imco adjusted index. Information for 2009-2012 is used. The independent variables represent objective insecurity, municipal and state institutional efficiency and confidence in the administration of state justice. We conclude that public insecurity has affected the attraction of investment and human talent and the competitiveness processes in most states.
\end{abstract}

Keywords: territorial competitiveness, public insecurity, panel data, gross fixed capital formation, human talent.

\section{Resumen}

El objetivo de esta investigación es determinar el impacto de la inseguridad pública y la violencia en la competitividad de las empresas instauradas en las entidades federativas, a partir de la definición del Instituto Mexicano para la Competitividad (Imco). Se aplica panel de datos a tres modelos con diferentes variables dependientes: formación bruta de capital fijo, talento humano e índice Imco ajustado. La información que se utiliza corresponde al periodo 2009-2012. Las variables independientes representan inseguridad objetiva, eficiencia institucional municipal y estatal, así como confianza en la procuración de justicia estatal. Se concluye que la inseguridad ha impactado en los procesos de inversión, de la formación y la atracción de talento humano en la mayoría de las empresas de las entidades federativas.

Palabras clave: competitividad territorial, inseguridad pública, panel de datos, formación bruta de capital fijo, talento humano.

* Universidad de Guadalajara, correo-e: soriaromo@yahoo.com.mx 


\section{Introducción}

Funcionarios, políticos, empresarios y ciudadanos en general afirman que la inseguridad del país es un elemento inhibidor de la inversión, por ende de la creación de empleos y del crecimiento económico. Es decir, se cree y se constata que ésta es un factor que impacta negativamente la competitividad de las empresas, las ciudades, las entidades federativas, las regiones y el país. Sin embargo, no existen estudios que prueben con rigor estadístico la existencia de esta relación, aunque en los últimos años se han escrito diversos trabajos que relacionan el crecimiento económico con la inseguridad tanto en el país como en otras naciones (Imco, 2006; González-Andrade, 2014; Robles et al., 2013; Villamarín, 2011; VerdugoYepes et al., 2015).

En este contexto, el objetivo del presente trabajo es probar empíricamente la relación entre la competitividad de las entidades federativas mexicanas y la violencia, la inseguridad pública y el buen o mal funcionamiento institucional prevaleciente en éstas. Para lo anterior se aplica el método de panel de datos, utilizando información para el periodo 20092012. Es conveniente enfatizar, para evitar confusiones, que el objeto de estudio son las entidades federativas mexicanas. El estudio de la relación entre competitividad y violencia e inseguridad en los municipios o las ciudades, bajo el enfoque aquí desarrollado, aunque es un tema muy interesante y urgente, está aún por realizarse.

El presente trabajo se divide en cinco apartados. En el primero se exponen algunos conceptos sobre la competitividad y se determina la definición a utilizar. En el siguiente apartado denominado "estado del arte" se debaten los principales trabajos desarrollados con respecto al tema, posteriormente se plantea el modelo, seguido por los resultados y, en la parte final, se manifiestan las conclusiones y las líneas de investigación a seguir. Se destaca que la violencia y la inseguridad afectan los procesos de la formación de capital y la generación y la atracción de talento humano en la mayoría de las entidades federativas.

\section{Competitividad territorial ${ }^{1}$}

La competitividad territorial es un concepto que se aplica para estudiar dicho fenómeno en municipios, ciudades, regiones, entidades federativas y naciones como un todo. El Imco utiliza este enfoque al desarrollar sus análisis en las diferentes demarcaciones. En lo que respecta a las entidades federativas, el "Índice de Competitividad Estatal" elaborado por el Imco

\footnotetext{
${ }^{1}$ Este apartado amplía el contenido de Soria (2013: 328-329).
} 
(2014: 154) "mide la capacidad de las entidades federativas para atraer y retener talento e inversiones". Para fines del presente trabajo se retoma esta conceptualización básica, a la que se le han añadido un conjunto de atributos. En 2007, el Imco (2007: 41) señaló:

La sostenibilidad es garante de las circunstancias y coyunturas que ofrecerá la región no sólo en los meses siguientes, sino, al menos, en el medio plazo. Este es un aspecto fundamental para la toma de decisiones económicas orientadas a la inversión. En sí mismo, el término sostenibilidad encierra gran parte del significado de lo que es e implica la competitividad.

Cabrero et al. (2007: 4-5) refuerzan lo anterior al señalar:

una ciudad competitiva no es aquélla que solo logra captar inversiones por sus bajos costos de mano de obra, sino es aquélla capaz de crear mejores condiciones capacitando más a la fuerza de trabajo, elevando la productividad y ofertando mejores condiciones urbano-ambientales e institucionales, ya sea en términos de infraestructura básica y de comunicaciones, como en calidad de los servicios especializados, innovaciones tecnológicas, medio ambiente sustentable, instituciones transparentes, calidad en las regulaciones, combate a la inseguridad y promoción de la cohesión social, es decir, mejores condiciones generales para la producción económica y para el desarrollo de la vida social.

La competitividad es un conjunto de elementos en los que la inclusión del abatimiento de las inequidades sociales se convierte en una palanca fundamental para la atracción de inversión y oportunidades (Cabrero et al., 2007: 4-5). Lever y Turak, 1999 citados en Sobrino (2005: 145) refieren el siguiente concepto, aplicado en este caso a las ciudades:

El concepto de competitividad urbana alude a la capacidad de una ciudad para insertarse en los mercados nacionales y foráneos, y su relación con el crecimiento económico local y el incremento en la calidad de vida de sus residentes. Otra definición de competitividad urbana es el grado en el cual las ciudades pueden producir bienes y servicios para los mercados regional, nacional e internacional, aumentando, de manera paralela, el ingreso real y la calidad de vida de la población y procurando un desarrollo sostenible.

Para fines del presente trabajo y sobre la base de los conceptos citados, se destacan algunas características que permitirán determinar una definición de la competitividad territorial (Soria y López Portillo, 2014: 86), la cual se define como la capacidad de atraer y retener inversiones y recursos de diversa naturaleza, destacadamente el talento humano. Dicha atracción debe ser sostenible y compatible con el equilibrio ecológico y del medio ambiente. El logro de la competitividad territorial no es un fin en sí mismo, sino que se concibe como un vehículo para generar empleo con salarios decentes, elevar el ingreso real y la calidad de vida de la po- 
blación, disminuir la pobreza y la equidad entre los residentes del territorio por medio de la inclusión y la cohesión social.

En el presente trabajo se busca aplicar el anterior concepto de competitividad territorial a las entidades federativas mexicanas. Al respecto de la definición anterior, se plantea el estado del arte, para posteriormente presentar el modelo de panel de datos como instrumento de análisis empírico.

\section{El estado del arte}

En este apartado se reseñan trabajos en dos vertientes: $a$ ) los que analizan la competitividad territorial relativa de los municipios, las ciudades o las entidades federativas y los que relacionan la inseguridad y la violencia con los niveles de crecimiento económico o sus efectos en diversos sectores de la actividad económica.

\subsection{Competitividad en ciudades y municipios}

Se revisan tres tipos de trabajos: los que estudian los municipios, los que analizan la ciudad — entendida como zona conurbada — y, finalmente, los que estudian regiones y entidades federativas. ${ }^{2}$ Entre los primeros destaca el de Quijano (2007) aplicado al estado de Sonora. El autor construye un índice de competitividad con componentes económicos, sociales, ambientales y globales. En el mismo sentido se encuentra el trabajo de Pérez et al. (2011), relativo al estado de Campeche, quienes construyen un índice de competitividad municipal que postulan es aplicable a cualquier municipio del país utilizando siete factores: los recursos humanos, la calidad de vida, las condiciones de la economía, la infraestructura, el ambiente de negocios y la eficiencia del gobierno.

Gutiérrez-Casas (2007), propone un enfoque que consiste en una versión revisada del enfoque del potencial de desarrollo, expuesto originalmente por Biehl (1986). Dicho modelo "permite la caracterización de las regiones a partir de factores de potencialidad y factores limitantes del desarrollo, y proporciona un sistema de clasificación regional apropiado para la definición y aplicación de políticas regionales que propicien el desarrollo" (Gutiérrez-Casas, 2007: 8), y se aplica a los municipios del estado de Chihuahua. Este autor concluye que a partir de su modelo es posible mostrar los claros desequilibrios territoriales y desigualdades socioeconómicas entre los municipios de Chihuahua, así como diferenciar

${ }^{2}$ Para un desarrollo más detallado de este tópico, véase Soria (2013). 
paquetes de políticas públicas destinadas a regiones atrasadas y vulnerables, y políticas orientadas a regiones avanzadas.

Existe otra vertiente de análisis conocida como "estudios sobre la ciudad", definida como un conjunto de municipios que integran zonas metropolitanas. Un trabajo pionero es el de Cabrero et al. (2005), que los mismos autores continuaron en 2007. En ambos estudios se consideran las 60 zonas metropolitanas más importantes del país en cuanto a población y generación de riqueza, en las cuales se integran un total de 225 municipios, representando a cada estado de la república. Los autores utilizan cuatro tipos de componentes: a) económico; $b$ ) urbano-ambiental; c) socio-demográfico y d) institucional.

De manera independiente, Sobrino (2005) reseña los indicadores más utilizados en la literatura y su ejemplificación con el caso mexicano. Analiza específicamente 39 ciudades del país y utiliza el Valor Bruto de la Producción para generar un índice de posición competitiva de las ciudades. En su trabajo el autor cita dos estudios previos (Sobrino, 2002 y 2003) como antecedentes.

El Imco (2007 y 2012) ha generado cuatro estudios de gran valor sobre el tema. En 2007 se incluyen 55 ciudades o zonas metropolitanas y en 2012 se amplía a 77 ciudades que concentran más de 50\% de la población y más de 70\% del PIB nacional. Ambos índices utilizan 60 indicadores, categorizados en 10 subíndices que son colectivamente exhaustivos y mutuamente excluyentes. Cada subíndice investiga una dimensión distinta de la competitividad (Imco, 2012). En la tabla 1 se presenta una comparación de los subíndices. Como se observa, el gran cambio entre 2007 y 2012 se centra en la inclusión del "sistema político estable y funcional" y el acotamiento de mercados de factores por mercado laboral, lo que resalta la importancia que el Imco le da a este tema, y la sustitución de "sectores económicos con potencial" por "sectores de innovación y sofisticación”.

En cuanto a la competitividad regional, Cabrero y Barbarín-Rodríguez (2011), basados en trabajos previos del Centro de Investigación y Docencia Económicas (CIDE) y en el índice desarrollado por dicha institución, dividen al país en cinco regiones socioeconómicas (noroeste, noreste, centro-occidente, centro y sureste), y se llevan dicha regionalización a nivel municipal, utilizando su perfil de competitividad, definido de acuerdo con el grado de penetración de elementos modernos en su industria, comercio y servicios y en el liderazgo que ejercen las ciudades en su región.

Como resultado del análisis, se identificaron los centros urbanos más dinámicos, de acuerdo con su competitividad. Se encontró que nueve de los 10 centros urbanos más competitivos también son los más dinámicos y los que tienen un liderazgo económico regional alto (Aguascalientes, 


\section{Tabla 1}

Comparativo de subíndices en los estudios del Imco (2007 y 2012)

\begin{tabular}{|c|c|}
\hline $\begin{array}{l}\text { Indice de Competitividad en Zonas Urbanas } \\
2007\end{array}$ & Indice de Competitividad Urbana 2012 \\
\hline a) Sistema de derecho confiable y objetivo & Mismo subíndice \\
\hline b) Manejo sustentable del medio ambiente & Mismo subíndice \\
\hline c) Sociedad incluyente, preparada y sana & Mismo subíndice \\
\hline \multirow[t]{2}{*}{ d) Economía dinámica e indicadores estables } & d) Economía estable \\
\hline & e) Sistema político estable y funcional \\
\hline f) Mercados de factores eficientes & f) Mercado laboral \\
\hline g) Sectores precursores de clase mundial & g) Infraestructura \\
\hline h) Gobierno eficiente y eficaz & h) Gobiernos eficientes y eficaces \\
\hline $\begin{array}{l}\text { i) Aprovechamiento de las relaciones interna- } \\
\text { cionales }\end{array}$ & Mismo subíndice \\
\hline j) Sectores económicos con potencial & i) Sectores de innovación y sofisticación \\
\hline
\end{tabular}

Fuente: Imco (2007 y 2012).

Ciudad de México, Ciudad Juárez, Guadalajara, Monterrey, Puebla, Querétaro, Saltillo y San Luis Potosí) (Cabrero y Barbarín-Rodríguez, 2011). Finalmente, el análisis de estos autores permite definir estrategias de generación de competencias específicas al perfil, grado de competitividad y liderazgo de cada ciudad, y por otro lado, identificar políticas públicas exitosas implementadas, lo que posibilita su adaptación a ciudades con un perfil similar.

Hasta este punto, se han reseñado trabajos relacionados con la competitividad tanto municipal como de ciudades como antecedentes. Sin embargo, el objeto de estudio del presente artículo es la relación entre la inseguridad y la violencia prevaleciente en las entidades federativas en México y su competitividad, mediado por la calidad del funcionamiento institucional, por lo que el estado del arte en este sentido se presenta en el siguiente apartado.

\subsection{El impacto de la violencia y la inseguridad pública en la actividad económica, con énfasis en las entidades federativas}

En los últimos años se ha desarrollado una creciente literatura que busca responder la pregunta ¿Cuál es la relación entre la violencia, la inseguridad pública y el crecimiento económico? o, ¿Cuál es el impacto de la inseguridad y la violencia en la actividad económica en los sectores económicos específicos? La presente sección reseña algunos de los principales avances en este aspecto. 
En primer lugar se encuentra el trabajo de Villamarín (2011), desarrollado para Colombia. Su principal objetivo es "calcular la pérdida de bienestar asociada con una mayor mortalidad generada por los homicidios en el periodo 1990-2005, teniendo en cuenta la heterogeneidad geográfica y demográfica de la violencia homicida" también estudia "la relación entre la violencia y las condiciones y posibilidades de desarrollo" (Villamarín, 2011: 1). La autora encuentra una relación positiva entre violencia y PIB per cápita y señala:

la relación entre violencia y desarrollo es compleja, ya que el crecimiento económico, es posible en medio de altos índices de violencia. Pero al mismo tiempo, precisamente por esto la violencia resulta más costosa que en países con economías menos dinámicas y pone freno a lo que, en potencia, sería un crecimiento aún mayor (Villamarín, 2011: 42).

Otro trabajo, pero aplicado al caso de las entidades federativas mexicanas, señala que el bajo crecimiento de la economía mexicana se puede explicar por el aumento de la delincuencia (Feliz, 2012: 222): "El crimen impone costos económicos importantes: pérdidas de vidas, disrupción de las actividades productivas, menos ahorro e inversión, estrés, y el enfoque de esfuerzos públicos y privados hacia actividades de seguridad".

Para lo anterior utiliza la teoría de crecimiento exógeno desarrollada por Solow y la teoría del crimen de Becker. De acuerdo con dicho modelo: "la actividad criminal reduce el producto de la economía directamente debido a que reduce el tiempo que se asigna a la producción e indirectamente reduciendo la inversión” (Feliz, 2012: 222).

Con este modelo se analiza la interacción del crecimiento económico y el crimen en las entidades federativas de México durante el periodo 2003-2010. Feliz (2012: 225) admite que el modelo puede no ser el apropiado a este propósito ya que supone una economía cerrada, "en cambio México es una economía abierta y sus estados federales tienen mercados de productos y factores integrados".

El autor encuentra una relación negativa, estadísticamente robusta, entre el crecimiento económico y la tasa de homicidios que se emplea como indicador de la actividad criminal, pues se observa una reducción de la tasa de crecimiento del PIB per cápita anual del $0.21 \%$, lo cual resulta en una "pérdida acumulada de $1.5 \%$ en el periodo 2003-2010, equivalente a la tercera parte del crecimiento observado en el periodo" (Feliz, 2012: 240).

Otro trabajo que analiza esta relación es el de Robles et al. (2013: 1920), referido a México. Los autores parten del supuesto de que: 
la violencia no tiene necesariamente un efecto lineal sobre el desempeño económico. Debajo de cierto umbral es posible que las empresas internalicen sus costos a través de pagos de derechos de piso, robos, tecnología para incrementar su protección (como cámaras en los tráileres), rescates, costos de guardaespaldas, entre otros. Bajo estas condiciones, la violencia podría afectar marginalmente tanto la demanda como la oferta laboral. Las empresas más pequeñas podrían dejar de operar haciendo que la demanda laboral se contraiga.

Es de esperarse que, alcanzado este umbral de guerra, la violencia relacionada con el narcotráfico impacte profundamente el desempeño económico. Bajo estas condiciones, es factible que un número considerable de empresarios decidan cerrar sus negocios y migrar con sus familias a zonas más seguras. Cuando varias empresas deciden dejar de operar, esperaríamos observar una disminución en la producción total de productos y servicios, así como también en la inversión, y una fuerte contracción del PIB.

Para probar su hipótesis, los autores utilizan el consumo municipal de electricidad como proxy del PIB municipal como variable dependiente. Los homicidios se utilizan como variable representativa de la violencia. Se encontró efectivamente que aquellos municipios que observaron incrementos drásticos de la violencia entre 2006 y 2010 redujeron de forma importante su consumo de energía durante los años posteriores al tratamiento. Esto implica que el mencionado umbral efectivamente opera, aunque, señalan los autores, son necesarias futuras investigaciones que modelen y estimen de forma más sofisticada la relación entre violencia y resultados económicos.

Un ensayo adicional que relaciona la incidencia delictiva con el crecimiento económico a nivel de entidades federativas es el de GonzálezAndrade (2014). Como punto de partida señala que "los estudios relevantes al crimen y la inseguridad están apenas comenzando" (González-Andrade, 2014: 76). El autor postula una relación inversa entre PIBE per cápita y tasas de incidencia delictiva tanto del fuero común como del fuero federal y de los delitos de alto impacto. Realiza su estudio con información de un periodo de ocho años (2003-2010) mediante un análisis de panel de datos con efectos fijos. Examina dos modelos econométricos en los cuales introduce diversas variables de control como la inversión extranjera directa, la población ocupada con acceso a las instituciones de salud, los niveles de escolaridad, la población desocupada, los recursos distribuidos a través del Ramo 33, enfatizando los recursos del Fondo de Aportaciones para la Seguridad Pública de los Estados y del Distrito Federal.

Las variables que representan la incidencia delictiva son los delitos denunciados en los fueros común y federal (contra la salud, delitos que 
contravienen la ley federal de armas de fuego y explosivos, así como delitos patrimoniales). En el segundo modelo, además de las variables económicas, se investiga el efecto de las variables criminales de alto impacto: robos, lesiones dolosas, homicidios culposos y dolosos, violaciones o delitos sexuales y secuestros. El autor concluye que:

el modelo base desarrollado exhibe una baja, pero significativa relación entre el crecimiento económico regional medido en valor del producto estatal bruto per cápita y las variables de delincuencia y criminalidad, medidas en tasas de incidencia relativa de delitos de los fueros común y federal. De igual manera, los resultados obtenidos en el modelo alternativo indican que los delitos de alto impacto, tales como homicidios, robos, y lesiones dolosas ejercen un efecto negativo sobre el crecimiento económico regional (González-Andrade, 2014: 14).

Finalmente, el autor enfatiza la necesidad de incorporar nuevas variables a los modelos, tales como gastos en educación o sofisticar las técnicas de estimación incluyendo impactos rezagados de las variables, entre otras. De esta forma se podrá aumentar la capacidad de explicación de los modelos aquí propuestos y será posible obtener mejores resultados.

El trabajo más reciente sobre el tema corresponde a Verdugo-Yepes et al. (2015: 4). El trabajo tiene tres propósitos: a) investigar el efecto de la delincuencia en la actividad económica general en el periodo 1993-2012; b) descubrir las relaciones causales subyacentes; $c$ ) dar cuenta de la naturaleza dinámica de tales relaciones y $d$ ) explicar la naturaleza heterogénea de tales relaciones entre los diferentes estados mexicanos. Los autores utilizan el método de vector autoregresivo estructural. Utilizan variables, tales como la tasa de homicidios, el PIB estatal per cápita, los flujos de inversión extranjera directa y los flujos migratorios netos hacia las entidades federativas.

Los autores concluyen que para un estado típico, un choque de criminalidad induce una caída del 0.5 por ciento del PIB estatal per cápita, misma que persiste por un lapso de dos años y se disipa gradualmente en el tercer año, aunque en los estados más violentos el efecto de la disipación es más lento. Sin embargo, la respuesta no es uniforme en las diferentes entidades federativas, con un número importante de estados con respuestas y efectos en direcciones opuestas.

Como se observa en esta sección no se incluyen trabajos que relacionan directamente la competitividad con inseguridad pública, la violencia y el funcionamiento institucional. El tópico más cercano es el que estudia el impacto de la violencia y la inseguridad en el crecimiento económico. Una perspectiva alternativa de analizar este problema es el modelo que forma el núcleo del presente artículo y se presenta en el apartado posterior. 


\subsection{La delincuencia: caracterización y medición}

En esta sección se hace una breve reseña de los trabajos más relevantes relacionados con los intentos de caracterización y medición de la incidencia delictiva y que a la vez son la base para la selección de las variables sobre este fenómeno, incorporadas al modelo econométrico incluido en el presente trabajo.

El primero de ellos corresponde a Carbonell (2013: 2). El propósito de este autor es "establecer un panorama comparativo sobre la violencia en los municipios, que en su mayoría son predominantemente urbanos, de más de 100 mil habitantes". El análisis se hace en 212 municipios y de acuerdo con lo que se reporta respecto al tema en 2012. La comparación se realiza con base en un índice de incidencia delictiva, integrado por seis delitos; se ordenan los 212 municipios para cada uno de los seis delitos y para el índice de violencia en su conjunto.

Los seis delitos considerados se caracterizan por ser violentos e intencionales: homicidio doloso, secuestro, violación, lesiones dolosas, robo con violencia y extorsión. Se calculan tasas por cada 100,000 habitantes para cada uno de ellos. El índice se expresa sobre la base de 100 puntos y se pondera de la siguiente forma: a) 55 puntos para homicidio doloso; b) 22, para secuestro; c) 13, para violación; d) cuatro, para lesiones dolosas; e) tres, para robos con violencia, y f) tres, para extorsión (Carbonell, 2013: 6).

Esta ponderación responde a varios criterios: a) el reproche diferenciado que las leyes penales hacen a los distintos delitos; $b$ ) las condenas que en efecto se imponen a los autores de dichos delitos (dentro de los márgenes de mínima y máxima pena); c) la trascendencia objetiva del delito; d) la duración (promedio) del evento criminal; $e$ ) los perjuicios físicos, económicos y psicológicos que el delito causa a las víctimas directas e indirectas; $f$ ) la perdurabilidad del trauma causado por el evento criminal; $g$ ) las posibilidades de recuperación de la víctima y; $h$ ) el efecto que para el conjunto social tiene cada tipo de delito (Carbonell, 2013: 6). Para la elaboración del modelo econométrico se utiliza como variable independiente el índice de incidencia delictiva o índice de violencia propuesto por este autor.

El Centro de Investigación para el Desarrollo (CIDAC, 2013: 9) desarrolló un segundo trabajo. Este índice enfatiza los delitos que afectan más la percepción de inseguridad de la población y logran modificar sus patrones de conducta. Se basó en:

utilizar el impacto que genera cada delito sobre la percepción de inseguridad de los ciudadanos. De forma general, la percepción de inseguridad o "miedo al cri- 
men" se forma a partir de la información que el individuo recibió en momentos anteriores: victimización familiar, victimización personal, crímenes con violencia y delitos sobre la propiedad en su entidad. Con esta información, el individuo evalúa el impacto de un delito futuro en su vida y lo jerarquiza respecto a otros delitos.

Con este argumento, CIDAC establece un orden de mayor a menor impacto de ocho delitos: secuestro, homicidio doloso, lesión dolosa, extorsión, robo sin violencia a transeúnte, robo con violencia a transeúnte, robo con violencia de vehículo y robo sin violencia de vehículo. Como se observa, todos los delitos del índice de incidencia de Carbonell se encuentran incluidos en el índice de CIDAC.

Por su parte, México Evalúa (s/f: 2-3) desarrolló un tercer trabajo en este sentido, en éste se expone una serie de índices e indicadores sobre cuatro temas: a) La magnitud del fenómeno, es decir, cuántos delitos se cometen, cuántos se denuncian, cómo se comportan los delitos graves y la relevancia de medir el secuestro; $b$ ) la percepción respecto a la inseguridad, y qué tanto miedo se tiene a ser víctima de un delito; $c$ ) la respuesta del sistema de seguridad ante el fenómeno: la capacidad del Estado para capturar e imponer castigo al delincuente y $d$ ) el gasto: los recursos gubernamentales destinados al combate de la inseguridad y su efectividad para lograr tal objetivo. El estudio reseñado es un tratamiento sistemático de indicadores e índices para medir la evolución (o retroceso) del fenómeno de la violencia y la inseguridad, y pretende informar y servir de base para los tomadores de decisiones, la opinión pública y la sociedad.

A partir de esta revisión de diversas clasificaciones del delito, se seleccionó el índice Carbonell para su incorporación como variable independiente al modelo econométrico, base del presente trabajo.

\subsection{Un comentario sobre el funcionamiento institucional}

El modelo econométrico expuesto en el siguiente apartado contiene dos variables que representan el funcionamiento institucional de gobiernos estatales y municipales, relacionadas con el ambiente de negocios, es decir, las llamadas "condiciones externas" mencionadas por Rubio y Baz (2005), además de las variables que representan la (in)seguridad pública. La razón de su inclusión en el modelo es porque dicho "ambiente de negocios", cuando funciona adecuadamente, constituye un bien público. Esto implica que si la delincuencia es baja, si existe certidumbre acerca de la propiedad de los bienes de los ciudadanos, si se respetan sus derechos, si existe seguridad en las inversiones, si el hecho de iniciar un negocio y de registrarlo es rápido y a un costo razonable, entre otros factores; este bien público contribuye a generar e incrementar la competitividad de las 
empresas en la(s) localidad(es) donde se aplica. Por otra parte, cuando la política de seguridad pública es incapaz de cumplir los propósitos anteriores y las instituciones no funcionan adecuadamente, se genera un incremento en sus costos, se cancelan planes de inversión, se cierran los negocios y, por ende, se disminuye la competitividad de las empresas, entonces se trata de un "mal público".

En estas circunstancias, la delincuencia impacta negativamente tanto la estructura de costos como la rentabilidad de las empresas ya que éstas tienen que desviar recursos a la prevención o remediación de la inseguridad, que en condiciones "normales" pudieran dedicar a ofrecer productos en el mercado a precios más accesibles y a la reinversión, a la generación de empleo y al propio crecimiento de la empresa y de la economía. Como corolario, se desprende que las empresas ubicadas en las entidades federativas en las que prevalece una mayor delincuencia son más afectadas en su competitividad. Por las razones anteriores conviene incluir en el modelo, variables que representan el buen o mal funcionamiento institucional.

\section{Hipótesis, metodología y modelo}

De acuerdo con el "estado del arte" no existen trabajos que relacionan directamente la competitividad con violencia e inseguridad pública en las entidades federativas. El tema más cercano es el que aborda el impacto de la inseguridad en el crecimiento económico en el ámbito estatal (González-Andrade, 2014 y Verdugo-Yepes et al., 2015). Sin embargo, este enfoque es erróneo ya que la relación entre violencia e inseguridad pública con el crecimiento económico es una relación indirecta que se encuentra mediada por diversos elementos, entre otros, la dotación de recursos naturales, la infraestructura física existente y el desarrollo tecnológico que, a su vez, impactan en primera instancia la competitividad y la productividad territorial y, posteriormente, el crecimiento económico. Por lo anterior, antes de estudiar la relación violencia-inseguridad-crecimiento económico se debe abordar la relación violencia-inseguridadfuncionamiento institucional-competitividad. Lo cual es el objeto del presente artículo.

Se parte de la definición de Rubio y Baz (2005: 70-71):

La competitividad es la capacidad de una empresa para enfrentar exitosamente a sus contrapartes. Una empresa es productiva cuando ha desarrollado las condiciones internas que le permiten competir y las externas que le adicionan capacidad para reducir costos y competir de manera exitosa. La optimización de los procesos internos de las empresas son tarea de cada empresario, pero las condiciones generales de la economía son lo que los economistas llaman un "bien público", es decir, un beneficio del cual se puede beneficiar por igual una empresa chica y 
una grande, una persona modesta o una acaudalada [...] Las empresas más competitivas serán aquellas que tengan una estructura interna óptima con un entorno que propicie su capacidad competitiva.

Para fines de este trabajo las palabras clave de la anterior definición son las "condiciones externas", pues la delincuencia en contra de las empresas, producto de la inseguridad, la violencia y el mal funcionamiento de las instituciones, son parte de dichas condiciones.

Cuando la política y las instituciones, en este caso las relacionadas con la seguridad pública, son incapaces de proporcionar un adecuado clima de negocios, de combatir la delincuencia y la impunidad y de brindar certidumbre a empresas y ciudadanos, como reacción, los agentes económicos deben incurrir en el incremento de costos como instalar alarmas, alambrados, bardas más altas, sistemas de vigilancia más sofisticados y caros, pago de "piso" a la delincuencia, contratación de elementos de seguridad, entre otros costos adicionales. Por el lado de las ventas, los negocios reaccionan recortando horarios de operación, los propietarios dejan de asistir a sus empresas por temor a ser secuestrados y, finalmente, se cancelan inversiones y se cierran negocios. Las actividades mencionadas afectan las ventas y los ingresos de las empresas y por ende disminuyen su competitividad. En este punto se alcanza el "umbral de guerra" mencionado anteriormente (Robles et al., 2013).

Por otra parte, las "condiciones externas", relacionadas con la delincuencia, la inseguridad y la violencia y el buen o mal funcionamiento institucional, varían por entidad federativa, por lo que su impacto en las empresas y consumidores es directamente proporcional a la intensidad existente en dichas condiciones externas.

De las anteriores consideraciones se puede plantear hipotéticamente la existencia de una relación inversa entre incidencia delictiva, violencia y mal funcionamiento institucional y la baja de la competitividad en las empresas, vía el incremento de costos de las empresas y la baja en sus ingresos. De igual forma, complementando la hipótesis, se postula que dicho impacto es diferencial dependiendo de las condiciones de violencia, inseguridad y funcionamiento institucional prevalecientes en cada entidad federativa.

El hecho de que las condiciones externas sean diferentes para cada entidad federativa permite introducir el aspecto territorial de la competitividad para probar empíricamente la relación entre inseguridad, violencia, funcionamiento institucional y competitividad estatal. El Imco (2014: 154) define la competitividad estatal como la "capacidad de una entidad federativa de atraer y retener inversión y talento humano". Éste es un concepto sencillo, fácil de modelar y de estudiar empíricamente. 
Para probar lo anterior, se utiliza un modelo de panel de datos con información anual para las 32 entidades federativas, cubriendo el periodo de 2009 a 2012. Dicho lapso de análisis se determina de acuerdo con la información disponible y relativamente confiable. En lo posible se utiliza la información proveniente de la base de datos de Imco (2014), ya que se considera que ha sido homogeneizada y tratada por posibles sesgos y lagunas originadas por su diferente origen. Para la información no disponible en la base del Imco se utilizan las fuentes originales.

El concepto de competitividad mencionado da la pauta para establecer tres modelos, en función de la variable dependiente (VD) de los mismos. En el primero de ellos, la VD es la formación bruta de capital fijo (FBCF) que representa todos los activos, infraestructura, instalaciones fijas, maquinaria y equipo (y por ende innovación y avance tecnológico), entre otros factores productivos que se agregan al stock de capital, de un país o región determinada en un periodo definido. La FBCF representa la inversión en la definición del Imco. La FBCF no sólo contribuye al crecimiento y al ciclo económico en el periodo en que se efectúa, sino que determina la capacidad y la calidad del crecimiento en el mediano y el largo plazo (CEPAL, 2015: 17).

De igual forma, la FBCF está directamente relacionada con la competitividad y productividad de la economía. Por lo anterior, si la inseguridad y la delincuencia afectan negativamente la FBCF, también perjudica la competitividad, la productividad y, por ende, la capacidad de crecimiento de una región o de un país tanto en el año en que se contrae la inversión como en los periodos subsiguientes, es decir, en el mediano y el largo plazo. Este es precisamente uno de los impactos que se mide en el presente artículo de manera comparativa entre las entidades federativas en México.

Una segunda VD consiste en el talento humano (TH) que se agrega a una economía en un periodo determinado. El TH está relacionado con la educación. De acuerdo con Briceńo-Mosquera (2011: 47):

La educación es un eje fundamental del desarrollo económico de una sociedad. Sobre ella recae en gran medida la posibilidad de que los países logren incrementar sus índices de innovación, productividad y crecimiento económico, permitiéndoles disminuir los niveles de pobreza e inequidad. La educación, además de generar beneficios individuales, también genera externalidades sociales en la medida que permite una mayor cohesión social y beneficios intergeneracionales. Coadyuva en la creación de ingresos futuros, dado que las personas adquieren conocimientos y desarrollan habilidades que les permiten incrementar sus capacidades productivas y de cohesión social, y de esta forma alcanzar un mayor bienestar. 
La educación, su nivel y calidad, la capacitación y la experiencia laboral de los recursos humanos constituyen la base del capital humano que impacta directamente la competitividad y la productividad económica. Se reconoce que las personas más educadas tienen mayores ingresos. Esto implica que los profesionistas tienden a recibir mayores ingresos porque tienen mayores "conocimientos útiles en el mercado de trabajo donde se presenta una oferta limitada" (Capocasale-Bruno, 2000: 78). Para fines del presente artículo, se toma la variable talento humano $(\mathrm{TH})$, definida por el Imco (2014) como la proporción de profesionistas entre las personas mayores de 25 años viviendo en una entidad federativa en un ańo determinado.

La tercer VD es una versión ajustada del ICE del Imco (2014). Como ya se señaló, este índice se integra por 10 subíndices (Imco, 2014; Soria y López Portillo, 2014). En el presente ejercicio, y para evitar problemas de especificación y multicolinearidad se eliminan las variables relacionadas con la incidencia delictiva del subíndice "Sistema de derecho confiable y objetivo". De igual forma del subíndice "Gobiernos eficientes y eficaces" se excluyen las variables que se integran en este modelo como independientes (registro de una propiedad y apertura de un negocio). La VD que se denomina Imco ajustado resta la aportación de ambos conjuntos de variables de los subíndices mencionados. Este tercer modelo se considera como una confirmación, por la vía estadística, de la medición de la competitividad estatal hecha por el Imco (2014).

Los tres modelos incorporan cuatro variables independientes y una de control, mismas que son comunes para todos ellos (las siglas entre paréntesis es el nombre de la variable en el modelo):

a) Índice de violencia (lniv). Índice de violencia, se toma el propuesto por Carbonell (2013), explicado en líneas anteriores. Representa la violencia objetiva, es decir, aquella que contienen los registros administrativos y no contemplan la cifra negra. La fuente es el Secretariado Ejecutivo del Sistema Nacional de Seguridad Pública.

b) Cifra negra (Incifranegra). Son los delitos del fuero común cometidos que no se denuncian o en los cuales no se inicia averiguación previa. Representa la confianza de la ciudadanía en el sistema de procuración de justicia a nivel estatal. Se toman los datos proporcionales. Esta información complementa la contenida en la variable Índice de violencia. La fuente es la Encuesta Nacional de Victimización y Percepción sobre Seguridad Pública del Inegi para varios años.

c) Días para registrar una propiedad (Indiasregpprop). Son los días que dura el registro público de la propiedad en realizar dicha actividad. 
Dado que esta agencia es parte del gobierno estatal, en estos modelos representa la eficiencia en el funcionamiento del poder ejecutivo del gobierno estatal.

d) Costo de apertura de negocios (Incostapeneg). Éste se representa por el porcentaje de ingresos per cápita que le cuesta a un negocio el inicio de sus operaciones (licencia de construcción, licencia de funcionamiento, entre otros). Dado que estos trámites son municipales, esta variable representa la eficiencia en el funcionamiento de los gobiernos municipales.

e) Población $($ Inpob). Población total de la entidad federativa, se establece como variable de control.

Para las variables c), d), y e), se toman los años 2007, 2009, 2012 y 2014 y la fuente es el World Bank (2015).

En trabajos preparatorios del presente artículo, se experimentó con la variable independiente lndiascumcont, que mide el número de días que tarda un juzgado local en resolver el cumplimiento de un contrato y representa la eficiencia en el funcionamiento del poder judicial local. Sin embargo, resultó no significativa en los tres modelos y con el signo contrario al esperado, por lo que se decidió eliminarla de las especificaciones finales.

De acuerdo con la hipótesis planteada, el signo esperado de los coeficientes de todas las variables en todos los modelos es negativo, es decir, se espera una relación inversa. A esta afirmación se exceptúan lnpob, que como variable de control se plantea una relación directa. Con respecto a las variables dicotómicas de las entidades federativas se espera una relación inversa. El cálculo de los tres modelos se realiza tomando los logaritmos naturales de todas las variables continuas con el fin de que representen elasticidades.

En pruebas realizadas, aunque no incluidas por problemas de espacio, se dedujo que el modelo de efectos fijos es superior a la regresión agrupada y al modelo de efectos aleatorios. Las variables dicotómicas de las entidades federativas sí son significativas, mientras que las correspondientes a los periodos no lo son. Por otra parte, se utiliza una transformación logarítmica (Gujarati y Porter, 2010), ya que tiene diversas propiedades que lo hacen atractivo. En primer lugar, los coeficientes miden la elasticidad de la variable dependiente en relación con las independientes. Una segunda ventaja es que reduce tanto la asimetría como la heteroscedasticidad de las variables (Gujarati y Porter, 2010).

Por lo anterior, se llega a la conclusión de que el modelo a aplicar es logarítmico en panel de datos con efectos fijos y variables dicotómicas por entidad federativa. Además, se corrigió por correlación contemporánea, 
heteroscedasticidad y autocorrelación, siguiendo la técnica de Errores Estándar Corregidos para Panel (Panel Corrected Standard Errors o PCSE) recomendada por Aparicio y Márquez (2005). ${ }^{3}$

\section{Resultados}

En los cuadros 1, 2 y 3 se presentan los resultados de acuerdo al significado de las siguientes variables: a) lnfbcf: logaritmo natural de la formación bruta de capital fijo (FBCF); b) lniv: logaritmo natural del índice de violencia; c) Incifranegra: logaritmo natural de la cifra negra; d) Indiasregpprop: logaritmo natural de los días que dura el registro público de la propiedad en registrar una de ellas; e) Incostapeneg: logaritmo del costo de apertura de negocios; f) lnpob: logaritmo natural de la población; g) Intalento humano: representa el logaritmo natural del talento humano (TH) y; h) lnimcoajustado: es el logaritmo natural del ICE ajustado con las especificaciones señaladas anteriormente.

En los tres modelos, los coeficientes para las entidades federativas toman como base a Campeche, estado que tuvo un menor promedio en el índice de violencia durante todo el periodo (2009-2012). Dado que la variable dependiente es logarítmica y las entidades federativas son representadas por variables dicotómicas, los valores de sus coeficientes representan las diferencias promedio porcentuales de la variable dependiente para cada entidad, con respecto a la entidad base de comparación, en este caso, Campeche.

El primer modelo tiene como variable dependiente, el logaritmo de la formación bruta de capital fijo:

$$
\ln \left(\ln f b c f_{i}\right)=\beta_{0}+\beta_{1} \text { lniv }+\beta_{2} \text { Incifranegra }+\beta_{3} \text { Indiasregpprop }+\beta_{4}
$$
Incostapeneg $+\beta_{5}$ lncostcumcont $+\beta_{6}$ lnpob $+\sum \varphi_{j}$ stado $_{j}+u_{i}$ $, j=1, \ldots 31$ entidades federativas.

Donde $\varphi_{j}$ son las variables dicotómicas de los estados incluidos en la estimación, desde $j=1$ para el estado 1 , hasta la entidad 31. En el primer modelo $\varphi_{j}$ representa el impacto promedio porcentual en la $f b c f$ de las características y factores específicos a cada entidad federativa no incluidos en las variables independientes. Se enfatiza que este resultado se debe interpretar en relación con Campeche, como estado base. El signo esperado de las variables dicotómicas estatales es negativo. En la medida que el valor de $\varphi_{i}$ es mayor, una entidad federativa es más competitiva y

\footnotetext{
${ }^{3}$ La forma de llegar a esta técnica se detalla en Soria (2015), basado en Aparicio y Márquez (2005). Para el modelo de panel de datos se consultó a Gujarati y Porter (2010); Balgati (2009); Balgati (2010). La estimación se realizó en Stata 12 (Hamilton, 2006; Torres-Reyna, 2011).
} 


\section{Cuadro 1 \\ Modelo 1. Variable dependiente: $\operatorname{lnfbcf}$ \\ Resultados del modelo de regresión Prais-Winsten: "Errores \\ Estándar Corregidos para Panel” (PCSE)}

\begin{tabular}{|c|c|c|c|}
\hline Variable & Coeficiente & $z$ & ¿Signo correcto? \\
\hline $\operatorname{lniv}$ & -.0293925 & -1.17 & sí \\
\hline lncifranegra & -.2428747 & -0.98 & sí \\
\hline Indiasregpprop & -.0029535 & -0.36 & sí \\
\hline lncostapeneg & $-.1039611^{* * *}$ & -4.17 & sí \\
\hline Lnpob & $5.157299^{* * *}$ & 6.34 & sí \\
\hline Coahuila & $-0.33146^{* * *}$ & -10.16 & sí \\
\hline Baja California Sur & $-0.51986^{* * *}$ & -2.40 & si \\
\hline Chihuahua & $-0.91944^{*}$ & -4.34 & sí \\
\hline Aguascalientes & $-3.24304^{* * *}$ & -11.98 & sí \\
\hline Nayarit & $-3.34568^{* * *}$ & -15.55 & sí \\
\hline Quintana Roo & $-3.60949^{* * *}$ & -9.50 & sí \\
\hline Tlaxcala & $-3.95726^{* * *}$ & -13.77 & sí \\
\hline Zacatecas & $-4.49693^{* * *}$ & -9.73 & sí \\
\hline Durango & $-4.79401^{* * *}$ & -8.96 & sí \\
\hline Querétaro & $-4.99848^{* * *}$ & -7.75 & sí \\
\hline Michoacán & $-5.31997^{* * *}$ & -8.81 & sí \\
\hline Tabasco & $-5.40677^{* * *}$ & -6.93 & sí \\
\hline Yucatán & $-5.61180^{* * *}$ & -8.14 & sí \\
\hline Sonora & $-6.55662^{* * *}$ & -6.98 & sí \\
\hline San Luis Potosí & $-6.70242^{* * *}$ & -7.42 & sí \\
\hline Sinaloa & $-6.98763^{* * *}$ & -7.27 & sí \\
\hline Hidalgo & $-7.02412^{* * *}$ & -7.55 & sí \\
\hline Chiapas & $-7.02560^{* * *}$ & -7.39 & sí \\
\hline Baja California & $-7.30873^{* * *}$ & -6.78 & sí \\
\hline Tamaulipas & $-7.47241^{* * *}$ & -6.77 & sí \\
\hline Guerrero & $-8.31380^{* * *}$ & -7.48 & sí \\
\hline Nuevo León & $-8.43702^{* * *}$ & -6.11 & sí \\
\hline Oaxaca & $-8.89400^{* * *}$ & -7.31 & sí \\
\hline México & $-9.23813^{* * *}$ & -6.99 & sí \\
\hline Colima & $-9.36969^{* * *}$ & -8.22 & sí \\
\hline Guanajuato & $-9.96181^{* * *}$ & -6.56 & sí \\
\hline Puebla & $-10.40162^{* * *}$ & -6.65 & sí \\
\hline Distrito Federal & $-10.78152^{* * *}$ & -5.70 & sí \\
\hline Jalisco & $-10.98099^{* * *}$ & -6.24 & sí \\
\hline Veracruz & $-11.28005^{* * *}$ & -6.32 & sí \\
\hline Morelos & $-14.29030^{* * *}$ & -6.10 & sí \\
\hline constante & $-57.100371^{* *}$ & -5.44 & sí \\
\hline $\mathrm{N}=128$ & No. grupos $=32$ & \multicolumn{2}{|c|}{$\mathrm{R} 2=0.9956$} \\
\hline
\end{tabular}

*significativo al $10 \%$; **significativo al $5 \%$; significativo al $1 \%$.

Fuente: elaboración propia, cálculos hechos con Stata 12. 


\section{Cuadro 2}

Modelo 2. Variable dependiente: Intalentohumano. Resultados del modelo de regresión Prais-Winsten, "Errores Estándar Corregidos para Panel” (PCSE)

\begin{tabular}{|c|c|c|c|}
\hline Variable & Coeficiente & $z$ & ¿Signo correcto? \\
\hline $\operatorname{lniv}$ & -.0253194 & -1.37 & sí \\
\hline lncifranegra & -.0213577 & -0.36 & sí \\
\hline Indiasregpprop & -.0091845 & -1.47 & sí \\
\hline lncostapeneg & -.0159908 & -1.18 & sí \\
\hline lnpob & $1.482199^{* * *}$ & 5.91 & sí \\
\hline Baja California Sur & $0.44975^{* * *}$ & 6.30 & no \\
\hline Chihuahua & $0.36827^{* * *}$ & 4.46 & no \\
\hline Coahuila & $-0.38720^{* * *}$ & -22.53 & sí \\
\hline Aguascalientes & $-0.46520^{* * *}$ & -5.44 & sí \\
\hline Quintana Roo & $-0.59691^{* * *}$ & -5.05 & sí \\
\hline Nayarit & $-0.60596^{* * *}$ & -9.01 & sí \\
\hline Tlaxcala & $-0.70245^{* * *}$ & -7.17 & sí \\
\hline Zacatecas & $-1.16456^{* * *}$ & -8.07 & sí \\
\hline Michoacán & $-1.19255^{* * *}$ & -6.50 & sí \\
\hline Durango & $-1.20942^{* * *}$ & -7.41 & sí \\
\hline Querétaro & $-1.26019^{* * *}$ & -6.34 & sí \\
\hline Yucatán & $-1.43784^{* * *}$ & -6.74 & sí \\
\hline Tabasco & $-1.53160^{* * *}$ & -6.47 & sí \\
\hline Sinaloa & $-1.68076^{* * *}$ & -5.77 & sí \\
\hline Chiapas & $-1.68855^{* * *}$ & -5.81 & sí \\
\hline Sonora & $-1.69775^{* * *}$ & -5.94 & sí \\
\hline Baja California & $-1.86734^{* * *}$ & -5.72 & sí \\
\hline San Luis Potosí & $-1.89970^{* * *}$ & -6.85 & sí \\
\hline Tamaulipas & $-1.97590^{* * *}$ & -5.87 & sí \\
\hline Hidalgo & $-2.18605^{* * *}$ & -7.65 & sí \\
\hline Colima & $-2.20423^{* * *}$ & -6.41 & sí \\
\hline Nuevo León & $-2.31414^{* * *}$ & -5.47 & sí \\
\hline Guerrero & $-2.41716^{* * *}$ & -7.12 & sí \\
\hline Oaxaca & $-2.67473^{* * *}$ & -7.14 & sí \\
\hline México & $-2.74078^{* * *}$ & -6.81 & sí \\
\hline Distrito Federal & $-2.93231^{* * *}$ & -5.05 & sí \\
\hline Puebla & $-3.23594^{* * *}$ & -6.73 & sí \\
\hline Jalisco & $-3.25142^{* * *}$ & -6.00 & sí \\
\hline Guanajuato & $-3.26911^{* * *}$ & -7.01 & sí \\
\hline Veracruz & $-3.45496^{* * *}$ & -6.30 & sí \\
\hline Morelos & $-4.31145^{* * *}$ & -6.01 & sí \\
\hline constante & $-17.90478^{* * *}$ & -5.23 & \\
\hline $\mathrm{N}=128$ & No. grupos $=32$ & \multicolumn{2}{|c|}{ R2 ajustada $=0.9770$} \\
\hline
\end{tabular}

*significativo al $10 \%$; ${ }^{* *}$ significativo al $5 \%$; significativo al $1 \%$.

Fuente: elaboración propia, cálculos hechos con Stata 12. 


\section{Cuadro 3}

Modelo 3. Variable dependiente: Inimcoajustado.

Resultados del modelo de regresión Prais-Winsten, "Errores Estándar Corregidos para Panel” (PCSE)

\begin{tabular}{|c|c|c|c|}
\hline Variable & Coeficiente & $z$ & ¿Signo correcto? \\
\hline $\operatorname{lniv}$ & -.0026506 & -0.28 & sí \\
\hline lncifranegra & -.023827 & -0.39 & sí \\
\hline lndiasregpprop & -.0016276 & -0.75 & sí \\
\hline lncostapeneg & -.0079236 & -0.94 & sí \\
\hline Lnpob & $.7585392^{* * *}$ & 8.66 & sí \\
\hline Baja California Sur & $0.38726^{* * *}$ & 12.83 & no \\
\hline Chihuahua & $0.30048^{* * *}$ & 9.91 & no \\
\hline Aguascalientes & $-0.05493^{*}$ & -2.07 & sí \\
\hline Quintana Roo & $-0.23760^{* * *}$ & -6.76 & sí \\
\hline Nayarit & $-0.27045^{* * *}$ & -11.91 & sí \\
\hline Coahuila & $-0.37980^{* * *}$ & -23.61 & sí \\
\hline Querétaro & $-0.46104^{* * *}$ & -6.83 & sí \\
\hline Tlaxcala & $-0.52729^{* * *}$ & -16.38 & sí \\
\hline Michoacán & $-0.57281^{* * *}$ & -9.06 & sí \\
\hline Zacatecas & $-0.58603^{* * *}$ & -12.31 & sí \\
\hline Yucatán & $-0.61342^{* * *}$ & -8.70 & sí \\
\hline Durango & $-0.68251^{* * *}$ & -13.50 & sí \\
\hline Sonora & $-0.77204^{* * *}$ & -8.01 & sí \\
\hline Chiapas & $-0.79344^{* * *}$ & -8.33 & sí \\
\hline Sinaloa & $-0.80217^{* * *}$ & -8.53 & sí \\
\hline Tabasco & $-0.85151^{* * *}$ & -11.17 & sí \\
\hline San Luis Potosí & $-0.88516^{* * *}$ & -9.57 & sí \\
\hline Baja California & $-0.92337^{* * *}$ & -8.60 & sí \\
\hline Tamaulipas & $-0.97646^{* * *}$ & -8.75 & sí \\
\hline Hidalgo & $-1.02617^{* * *}$ & -10.73 & sí \\
\hline Colima & $-1.03147^{* * *}$ & -9.23 & sí \\
\hline Nuevo León & $-1.03579^{* * *}$ & -7.32 & sí \\
\hline Distrito Federal & $-1.36017^{* * *}$ & -6.99 & sí \\
\hline México & $-1.41935^{* * *}$ & -10.52 & sí \\
\hline Guanajuato & $-1.42171^{* * *}$ & -8.91 & sí \\
\hline Guerrero & $-1.47285^{* * *}$ & -13.24 & sí \\
\hline Jalisco & $-1.54554^{* * *}$ & -8.39 & sí \\
\hline Puebla & $-1.54599^{* * *}$ & -9.48 & sí \\
\hline Oaxaca & $-1.55747^{* * *}$ & -12.58 & sí \\
\hline Veracruz & $-1.80971^{* * *}$ & -9.64 & sí \\
\hline Morelos & $-2.22249^{* * *}$ & -9.22 & sí \\
\hline constante & $-6.603057^{* * *}$ & -6.05 & \\
\hline $\mathrm{N}=128$ & No. grupos $=32$ & \multicolumn{2}{|c|}{$\mathrm{R}^{2}=0.9979$} \\
\hline
\end{tabular}

*significativo al 10\%; ${ }^{* *}$ significativo al 5\%; significativo al 1 .

Fuente: elaboración propia, cálculos hechos con Stata 12. 
cuando el valor del coeficiente es negativo y decreciente, dicho estado es más afectado por la violencia y la inseguridad. Para facilitar el análisis de los resultados, en los cuadros 1, 2 y 3 se ordenan los coeficientes de las entidades federativas de mayor a menor, es decir, las entidades se presentan de las más competitivas a las más negativamente impactadas por la inseguridad y la violencia.

Los resultados del primer modelo se presentan en el cuadro 1, donde la variable dependiente es $\ln f b c f$. En lo que respecta a las variables independientes, una de ellas (Incostapeneg), es significativa con 99\% de confianza. Las restantes variables independientes tienen el signo correcto: Iniv; Incifranegra y Indiasregpprop.

El resultado más importante del trabajo se relaciona con las variables dicotómicas. Todas las entidades federativas tienen el signo correcto y son significativas al 99\%, excepto Chihuahua, que es significativa al 90\%. Todas las entidades federativas son más impactadas que Campeche en sus procesos de inversión. Los estados menos afectados son Coahuila, Baja California Sur y Chihuahua con un promedio de 0,33, 0,52 y 0,92 veces, respectivamente, de impacto en sus procesos de formación bruta de capital fijo, con respecto a Campeche. En el otro extremo se encuentran Puebla, el Distrito Federal, Jalisco, Veracruz y Morelos que durante el periodo de análisis se vieron impactados 10,40; 10,78; 10,98; 11,28 y 14,29 más veces, respetivamente, que Campeche en su procesos de inversión. El resto de las entidades, de Aguascalientes a Puebla, recibieron un impacto de la violencia y la inseguridad de entre 3,24 y 9,96 veces más que Campeche.

El segundo modelo tiene como variable independiente el lntalentohumano y se representa en la siguiente ecuación:

$$
\begin{aligned}
& \ln (\text { Intalentohumano })=\beta_{0}+\beta_{1} \text { lniv }+\beta_{2} \text { Incranegra }+\beta_{3} \text { Indiasregpprop }+ \\
& \beta_{4} \text { lncostapeneg }+\beta_{5} \text { lncostcumcont }+\beta_{6} \operatorname{lnpob}+\sum \varphi_{j} \text { estadoj }+u i \\
& , j=1, \ldots 31 \text { entidades federativas. }
\end{aligned}
$$

Sus resultados se reportan en el cuadro 2. En este caso, las cuatro variables independientes tienen el signo correcto, aunque ninguna es significativa. En lo que respecta a las entidades federativas se encuentran resultados interesantes. Todos los estados tienen resultados significativos al 99\%. Dos entidades (Baja California Sur y Chihuahua) son más atractivos al talento humano que Campeche, lo que se refleja en el signo positivo de su coeficiente en 0,45 y 0,37 veces, respectivamente. Además existen cinco estados relativamente poco afectados: Coahuila, Aguascalientes, Quintana Roo, Nayarit y Tlaxcala con una afectación superior a Campeche en 0,$39 ; 0,47 ; 0,60 ; 0,61$ y 0,70 veces. En el otro extremo, las 
entidades más impactadas en sus procesos de atracción de capital humano por la inseguridad y la violencia son Puebla, Jalisco, Guanajuato y Veracruz que triplican dicho impacto en relación con Campeche. En el caso de Morelos, lo cuadruplica. El resto de los estados son impactados entre una y dos veces por el efecto de la violencia y la inseguridad en la atracción de talento humano.

El tercer modelo plantea como variable independiente el lnimcoajustado, cuya construcción fue explicada anteriormente. En este caso también se observa que las cuatro variables independientes tienen el signo correcto, aunque no son significativas.

$\ln \left(\right.$ Inimcoajustado $\left.{ }_{i}\right)=\beta_{0}+\beta_{1}$ lniv $+\beta_{2}$ Incifranegra $+\beta_{3}$ Indiasregpprop

$+\beta_{4}$ lncostapeneg $+\beta_{5}$ lncostcumcont $+\beta_{6} \ln p o b+\sum \varphi_{j}$ estado $+u i$ , $j=1, \ldots 31$ entidades federativas.

En lo que respecta a las variables dicotómicas de los estados, todas resultan significativas a 99\% de confianza. De nuevo, los estados de Baja California Sur y Chihuahua resultaron mejor posicionados que Campeche. Por otra parte, se observa un total de 17 entidades en las cuales el impacto en la variable dependiente es relativamente bajo (coeficiente negativo menor a 1). En el extremo opuesto, Jalisco, Puebla, Oaxaca y Veracruz tienen un coeficiente negativo mayor a 1,5 veces, y Morelos, la entidad menos competitiva duplica el impacto en lnimcoajustado de Campeche. Los resultados de este modelo se presentan en el cuadro 3. En los tres modelos, la variable de control observa el signo esperado y es significativa a 99 por ciento.

La información utilizada en este artículo comprende el periodo 20092012, por lo que los resultados de los modelos reportados son aplicables únicamente a dichos años. A pesar de este hecho, ya se pueden visualizar algunas tendencias de la violencia y la inseguridad en las entidades federativas que prevalecen hasta nuestros días. En primer lugar, en los tres modelos las entidades más competitivas son consistentemente: Baja California Sur, Coahuila, Chihuahua y Aguascalientes, seguidas por Quintana Roo a las que se debe agregar Campeche, que es la entidad de referencia, tanto en lo que se refiere a la atracción y retención de la inversión y al talento humano como en términos del ICE ajustado. En el extremo opuesto, las entidades que son menos competitivas de manera consistente en los tres modelos son Veracruz y Morelos, a los que se agregan Puebla, el Distrito Federal, Jalisco y Oaxaca, en función de la variable dependiente del modelo. Algunas de estas entidades, por ejemplo, Veracruz y Morelos son todavía en la actualidad destacadamente violentas e inseguras. Lo mismo se puede seńalar de Jalisco. 


\section{Conclusiones y futuras líneas de trabajo}

El presente trabajo analiza la relación entre inseguridad y violencia y competitividad en las entidades federativas en México, utilizando información para el periodo 2009-2012 y mediante la aplicación del método de panel de datos. La primera reflexión es que es un tema desconocido y novedoso tal como lo señalara González-Andrade (2014) y es necesario avanzar rápida y consistentemente en descifrar la relación entre la violencia, la inseguridad pública y su impacto en la competitividad territorial así como el crecimiento económico, tanto de las entidades federativas como de las ciudades mexicanas y del país en su conjunto vis a vis otras naciones.

En el trabajo se reflejan los claroscuros de esta relación y el estado del arte en cuanto a técnicas estadísticas y disponibilidad de información confiable. Por un lado, las variables independientes que representan violencia e inseguridad, así como las que representan el funcionamiento institucional de los gobiernos estatales y municipales en México y su capacidad de contrabalancear la violencia y la inseguridad resultan, en algunos casos, estadísticamente no significativas.

A pesar de lo anterior, y evaluando los resultados a partir de las variables dicotómicas que representan las entidades federativas en los modelos y que recogen sus características individuales y variables no observables, y considerando que son casi en la totalidad de las veces significativas a 99\%, tomando como base a Campeche, que fue la entidad menos insegura y violenta en el periodo 2009-2012, se considera que se muestra amplia evidencia del impacto negativo de la inseguridad pública sobre la competitividad de las entidades federativas.

Los trabajos reseñados en el subapartado 2.2. tratan de medir el impacto directo de la violencia y la inseguridad, ya sea como un porcentaje (reducción de la tasa de crecimiento del PIB per cápita anual de $0.21 \%$ según reporta Feliz (2012); reducción de forma importante de su consumo de energía (como variable proxy del PIB municipal) durante los años posteriores al impacto delincuencial (Robles et al., 2013); o según González-Andrade (2014: 104) "el modelo base desarrollado exhibe una baja, pero significativa relación entre el crecimiento económico regional medido en valor del producto estatal bruto per cápita y las variables de delincuencia y criminalidad", o lo señalado por Verdugo-Yepes et al. (2015), quienes concluyen que para un estado típico, un choque de criminalidad induce una caída de $0.5 \%$ del PIB estatal per cápita, por un lapso de dos ańos, y se disipa gradualmente en el tercer año.

Los resultados del presente artículo difieren, en el sentido que representan los impactos de la violencia, la inseguridad y el mal funcionamien- 
to institucional, en relación con la "mejor" entidad federativa en el periodo de estudio, que en este caso es Campeche. De esta manera, se detectan los estados más inseguros, lo cual permite señalar las entidades federativas en las que es necesario priorizar las estrategias para acotar la violencia y la inseguridad, y establecer programas de mejora institucional tanto a nivel estatal como municipal. Se considera que ésta es una perspectiva diferente de análisis que complementa los trabajos reseñados en el subapartado 2.2.

Una línea de investigación que se desprende del presente trabajo es sin duda estudiar la relación entre inseguridad y violencia y competitividad en las ciudades mexicanas, entendidas como áreas metropolitanas o zonas conurbadas, en virtud de que la delincuencia no reconoce fronteras intermunicipales, aunque también sería importante analizar este tema para los municipios como entidades autónomas y titular de las atribuciones de seguridad pública. Específicamente, es importante analizar el tema del "umbral de guerra" en las ciudades de México, es decir, en qué ciudades o áreas metropolitanas ya se ha rebasado el punto o umbral en el que las empresas ya no pueden internalizar los costos provocados por la violencia y la inseguridad, y se ven obligadas a limitar sus operaciones o de plano cerrar y abandonar la plaza; o cuáles ciudades se están aproximando a dicho "umbral de guerra". Esta problemáica requiere de una respuesta urgente, dada la situación prevaleciente en el país.

Finalmente, desde un punto de vista técnico, las diversas maneras en que se puede avanzar en la mejora de esta línea de investigación: a) trabajar un periodo más extenso; $b$ ) trabajar con variables latentes generadas por análisis factorial para evitar problemas de multicolinearidad; $c$ ) ampliar el problema de investigación de la relación entre inseguridad y competitividad territorial al impacto de la gestión pública sobre la competitividad, entre otros aspectos.

\section{Fuentes consultadas}

Aparicio-Castillo, Francisco Javier y Javier Márquez (2005), Diagnóstico y especificación de modelos panel en Stata 8.0, Centro de Investigación y Docencia Económicas, División de Estudios Políticos, Ciudad de México, México.

Balgati, Badi (2010), Econometric analysis of panel data, Wiley and Sons, Chichester, Reino Unido.

Balgati, Badi (2009), A companion to econometric analysis of panel data, Wiley and Sons, Chichester, Reino Unido. 
Biehl, Dieter (1986), The contribution of infrastructure to the regional development. Final report, Commission of the European Communities, Research report, Infrastructure Study Group, Luxemburgo, Luxemburgo.

Briceño-Mosquera, Andrea (2011), "La educación y su efecto en la formación de capital humano y en el desarrollo económico de los países", Apuntes del CENES, 30 (51), Universidad Pedagógica y Tecnológica de Colombia, Bogotá, Colombia, pp. 45-59.

Cabrero-Mendoza, Enrique y José Reynaldo Barbarín-Rodríguez (2011), "La diversidad de la competitividad de las ciudades mexicanas, perfiles y regiones contrastantes" en Rodolfo García del Castillo y Socorro Arzaluz-Solano (coords.), Gobiernos municipales: desarrollo, cohesión social y democracia participativa, Red de Investigadores en Gobiernos Locales, Ciudad de México, México, pp. 67-102.

Cabrero-Mendoza, Enrique, Isela Orihuela-Jurado y Alicia ZiccardiContigiani (2007), "Competitividad de las ciudades mexicanas. La nueva agenda de los municipios urbanos", Centro de Investigación y Docencia Económicas, México. <www.cide.edu/programas/Documento_Indice_Competitividad_Ciudades_ Mex_2007>, 29 de agosto de 2012.

Cabrero-Mendoza, Enrique, Isela Orihuela-Jurado y Alicia ZiccardiContigiani (2005), "Ciudades competitivas ciudades cooperativas: conceptos clave y construcción de un índice" en Carlos ArceMacías, Enrique Cabrero-Mendoza y Alicia Ziccardi-Contigiani (coords.), Ciudades del siglo XXI: ¿Competitividad o cooperación?, Centro de Investigación y Docencia Económicas y Miguel Ángel Porrúa, Ciudad de México, México, pp. 105-145.

Capocasale-Bruno, Alejandra (2000), “Capital humano y educación. Otro punto de vista”, Nueva Sociedad, núm. 165, Fundación Foro Nueva Sociedad, Buenos Aires, Argentina, pp. 73-84.

Carbonell, Miguel (2013), La violencia en los municipios de México 2012, Consejo Ciudadano para la Seguridad Pública y Justicia Penal A. C., Ciudad de México, México, <www.seguridadjusticiaypaz. org.mx>, 21 de enero de 2014. 
CEPAL (Comisión Económica para América Latina) (2015), Estudio económico de América Latina y el Caribe. Desafios para impulsar el ciclo de inversión con miras a reactivar el crecimiento, Naciones Unidas-CEPAL, New York, Estados Unidos de América.

CIDAC (Centro de Investigación para el Desarrollo) (2013), "8 Delitos Primero. Índice Delictivo CIDAC”, CIDAC, México, <www. cidac.org.>, 4 de septiembre de 2013.

Feliz, Raúl Aníbal (2012), “Crimen y crecimiento económico en México. Los estados federales en el periodo 2003-2010" en José Antonio Aguilar (ed.), Las bases sociales del crimen organizado y la violencia en México, Centro de Investigación y Estudios en Seguridad (CIES), Ciudad de México, México, pp. 221-245.

González-Andrade, Salvador (2014), "Criminalidad y crecimiento económico regional en México", Frontera Norte, 26 (51), El Colegio de la Frontera Norte, Tijuana, México, pp. 75-111.

Gujarati, Damoadar y Dawn Porter (2010), Econometría, McGraw Hill, Ciudad de México, México.

Gutiérrez-Casas, Luis Enrique, (2007), "Potencial de desarrollo y gestión de la política regional. El caso de Chihuahua”, Frontera Norte, 19 (2), El Colegio de la Frontera Norte, Tijuana, México, pp. 7-35.

Hamilton, Lawrence (2006), Statistics with Stata, Thomson, Belmont, California, Estados Unidos de América.

Imco (Instituto Mexicano para la Competitividad) (2014), "Índice de competitividad estatal 2015. Las reformas y los estados: la responsabilidad de las entidades en el éxito de los cambios estructurales", Imco, Ciudad de México, México, <http://imco.org.mx/ es/>, 18 de septiembre de 2014.

Imco (Instituto Mexicano para la Competitividad) (2012), "Índice de Competitividad Urbana 2012. El municipio: una institución diseñada para el fracaso. Propuestas para la gestión profesional de las ciudades", Imco, Ciudad de México, México, <http://imco. org.mx/es/>, 22 de agosto de 2013. 
Imco (Instituto Mexicano para la Competitividad) (2007), "Competitividad Urbana 2007”, Imco, Ciudad de México, México, <http:// imco.org.mx/es/>, 25 de agosto de 2015.

Imco (Instituto Mexicano de la Competitividad) (2006), "Situación de la competitividad en México", Imco, Ciudad de México, México, <http://imco.org.mx/es/>, 29 de julio de 2012.

Lever William F. e Ivan Turak (1999), "Competitive Cities: Introduction to the Review", Urban Studies, 36 (5-6), Sage Publishing, Londres, Reino Unido, pp. 791-793.

México Evalúa (s/f), "Indicadores para entender y monitorear la Seguridad Pública en México", México Evalúa, A. C., Ciudad de México, México, <www.mexicoevalua.org>, 23 de mayo de 2015.

Pérez-Canul, Carlos, Román Alberto Quijano-García y Mario Hernán Cetina-Pérez (2011), "La competitividad territorial del estado de Campeche", Ciencias Administrativas. Teoría y Praxis, 1 (7), Academia Mexicana de Ciencias Administrativas, Ciudad de México, México, pp. 167-185.

Quijano-Vega, Gil Arturo (2007), "La importancia de la competitividad económica en el desarrollo de los municipios sonorenses", Observatorio de la Economía Latinoamericana, 7 (7), Universidad de Málaga, Málaga, España, <www.eumed.net/cursecon/ecolat/ $\mathrm{mx} />, 15$ de agosto de 2012 .

Robles, Gustavo, Gabriela Calderón y Beatriz Magaloni (2013), "Las consecuencias económicas de la violencia del narcotráfico en México", Documento de trabajo núm. IDB-WP-426, Banco Interamericano de Desarrollo, Washington, Estados Unidos de América, pp. 49.

Rodríguez-Gómez, César Augusto (2008), "La competitividad en los municipios de México", documento de trabajo núm. 55, Centro de Estudios Sociales y de Opinión Pública, Cámara de Diputados, Ciudad de México, México, pp. 35.

Rubio, Luis y Verónica Baz (2005), El poder de la competitividad, Fondo de Cultura Económica y Centro de Investigación para el Desarrollo, Ciudad de México, México. 
Sobrino, Luis Jaime (2005), "Competitividad territorial: Ámbitos e indicadores de análisis", Economía, Sociedad y Territorio, Dossier especial, El Colegio Mexiquense, A. C., Toluca, México, pp. 123-183.

Sobrino, Luis Jaime (2003), Competitividad de las ciudades en México, El Colegio de México, Ciudad de México, México.

Sobrino, Luis Jaime (2002), "Competitividad y ventajas competitivas: revisión teórica y ejercicio de aplicación a 30 ciudades de México", Estudios Demográficos y Urbanos, 17 (2), El Colegio de México, Ciudad de México, México, pp. 311-363.

Soria-Romo, Rigoberto (2015), "El impacto de la inseguridad pública en la competitividad territorial: un estudio exploratorio de las entidades federativas", ponencia presentada al IX Congreso de la Red Internacional de Investigadores en Competitividad, 11-13 de noviembre, Puerto Vallarta, México.

Soria-Romo, Rigoberto y Carlos López Portillo-Tostado (2014), “Determinantes de la competitividad en las ciudades mexicanas: un análisis de panel con la base de datos Imco 2012” en José SánchezGutiérrez (coord.), La competitividad y los factores determinantes en la globalización, Red Internacional de Investigadores en Competitividad, Zapopan, Jalisco, México, pp. 77-100.

Soria-Romo, Rigoberto (2013), "Determinantes de la competitividad y de la inseguridad: el caso de los municipios de Jalisco" en José Sánchez-Gutiérrez, Juan Gaytán-Cortés, Guillermo VázquezÁvila, Juan A. Vargas-Barraza y Manuel Vázquez-Sandoval (coords.), La arquitectura financiera y desarrollo tecnológico para promover la competitividad, Ediciones de la Noche, Guadalajara, México, pp. 321-339.

Torres-Reyna, Oscar (2011), "Panel data analysis. Fixed and random effects (using Stata 10.x)", <http://dss.princenton.edu/training/>, 20 de agosto de 2015.

Verdugo-Yepes, Concepción, Peter Pedroni and Xingwei Hu (2015), "Crime and the economy in Mexican states: heterogeneous panel estimates (1993-2012)”, working paper núm WP/15/121, International Monetary Fund, Washington, Estados Unidos de América. 
Villamarín G., Adriana (2011), "Prevenir y calcular: una estimación de los costos de la violencia homicida en Colombia", documentos del Centro de Recursos para el Análisis de Conflictos (Cerac), Bogotá, Colombia, <www.cerac.org.co/>, 25 de noviembre de 2014.

World Bank (2015), "Doing Business. Midiendo Regulaciones para Hacer Negocios", Washington, United States of America, <www. doingbusiness.org/>, 3 de diciembre de 2015.

Recibido: 14 de enero de 2016.

Corregido: 6 de junio de 2016. Aceptado: 14 de febrero de 2016.

Rigoberto Soria Romo. Es doctor en estudios organizacionales por la Universidad Autónoma Metropolitana unidad Iztapalapa, México. Actualmente es profesor-investigador, adscrito al Departamento de Políticas Públicas, Centro Universitario de Ciencias Económico Administrativas, Universidad de Guadalajara. Es miembro del Sistema Nacional de Investigadores, nivel I, desde enero de 2006. Su línea de investigación actual es la relación entre la violencia y la inseguridad, así como la competitividad y los mecanismos de financiamiento de la seguridad pública local. Entre sus últimas publicaciones destacan, en coautoría: "Mecanismos de democracia directa en Jalisco, México: entre la contención, la simulación y la manipulación ciudadana. Cuatro estudios de caso", Telos, Revista de Estudios Interdisciplinarios en Ciencias Sociales, 18 (1), Universidad Rafael Belloso Chacín (URBE), Maracaibo, Venezuela, pp. 39-59 (2016); “¿Porque puede fracasar una política pública? El caso del Programa de Subsidio para la Seguridad Pública de los Municipios y Demarcaciones Territoriales del Distrito Federal", Revista de El Colegio de San Luis, Nueva Época, V (10), El Colegio de San Luis, San Luis Potosí, México, pp. 38-68 (2015); "Alternancia política y nueva gestión pública: un análisis comparativo, 1998-2008”, Región y Sociedad, XXV (56), El Colegio de Sonora, Hermosillo, Sonora, México, pp. 161-201 (2013); “Determinantes de la competitividad en las ciudades mexicanas: un análisis de panel con la base de datos Imco 2012" en José Sánchez Gutiérrez et al., La competitividad y los factores determinantes en la globalización, Red Internacional de Investigadores en Competitividad, Zapopan, Jalisco, México, pp. 77-100 (2014). 